\title{
Task- and location-switching effects on visual attention
}

\author{
JAMES F. JUOLA \\ University of Kansas, Lawrence, Kansas \\ Universidad Autónoma de Madrid, Madrid, Spain \\ and IPO Center for User-System Interaction, Eindhoven, The Netherlands \\ and \\ JUAN BOTELLA and ANTONIO PALACIOS \\ Universidad Autónoma de Madrid, Madrid, Spain
}

\begin{abstract}
In two experiments, we examined the effects of task and location switching on the accuracy of reporting target characters in an attentional blink (AB) paradigm. Single-character streams were presented at a rate of 100 msec per character in Experiment 1, and successive pairs of characters on either side of fixation were presented in Experiment 2. On each trial, two targets appeared that were either white letters or black digits embedded in a stream of black letter distractors, and they were separated by between zero and five items in the stream (lags 1-6). Experiment 1 showed that report of the first target was least accurate if it immediately preceded the second target and if the two targets were either both letters or both digits (task repetition cost). Report of the second target was least accurate if one or two distractors intervened between the two targets (the U-shaped $A B$ lag effect) and if one target was a letter and the other a digit (task switch cost). Experiment 2 added location uncertainty as a factor and showed similar effects as Experiment 1, with one exception. Lag 1 sparing (the preserved accuracy in reporting the second of two targets if the second immediately follows the first) was completely eliminated when the task required attention switching across locations. Two-way additive effects were found between task switching and location switching in the $\mathrm{AB}$ paradigm. These results suggests separate loci for their attentional effects. It is likely that the $\mathrm{AB}$ deficit is due mainly to central memory limitations, whereas location-switching costs occur at early visual levels. Task-switching costs occur at an intermediate visual level, since the present task switch involved encoding differences without changes in stimulus-response mapping rules (i.e., the task was character identification for both letters and digits).
\end{abstract}

The distribution of visual attention over natural environments has both spatial and temporal components. Attention helps us to identify objects against a background, as well as to follow these objects as they enter into events with causes and effects that unfold over time. Much research has shown that the distribution of spatial attention is determined by both endogenous processes (internal strategies related to task goals or instructions) and exogenous processes (reactions to external stimulus properties, such as contrast, motion, or sudden onsets; Briand \& Klein, 1987; Juola, Koshino, \& Warner, 1995; Müller \& Rabbitt, 1989; Posner, 1980; Wolfe, 1994). Similarly, when a series of events is experienced, either through

We acknowledge support from the General Research Fund of the University of Kansas and from Grants SAB1998-0161 to the first author and PB97-0012 to the second author from the Ministerio de Ciencia y Tecnología of Spain. Thanks are also due Maria Isabel Barriopedro for computer assistance with Experiment 1. We are grateful to Vincent Di Lollo, Mary Potter, Kimron Shapiro, Shui-I Shih, and an anonymous reviewer for helpful comments on an earlier version of this article. Correspondence concerning this article should be addressed to J. F. Juola, Department of Psychology, University of Kansas, 1415 Jayhawk Boulevard, Lawrence, KS 66045 (e-mail: juola@ku.edu). rapid changes, such as those that occur in sporting events, or through a succession of eye movements and fixations, some events appear to be selected for further processing to the relative exclusion of others, again due to the operation of both endogenous and exogenous processes (e.g., Norman \& Shallice, 1986).

In the laboratory, the temporal distribution of visual attention has been studied most intensively using procedures such as visual masking and the rapid serial visual presentation (RSVP) technique. In these procedures, two or more individual stimuli are presented to a single central locus on a display. If they occur in close succession (e.g., with a stimulus onset asynchrony [SOA] of less than about $60 \mathrm{msec}$ ), backward masking by visual integration or interruption processes can result in poor recognition of earlier items (e.g., Breitmeyer, 1984; Turvey, 1973). Slightly slower rates (e.g., an SOA of about $100 \mathrm{msec}$ ) ensure recognition of most items, but if the series contains more than about five items, memory consolidation and detailed report is possible for only a few of them. In order to study the temporal distribution of visual attention, RSVP tasks commonly define one or more items in a series as targets, by differentiating them in some way 
from the distractors, and the accuracy of the target report is the dependent measure. Although there is some evidence that identification of the first target (T1) can be negatively affected by the presence of a second target (T2) that immediately follows it (e.g., Broadbent \& Broadbent, 1987), the more interesting finding is called an attentional blink (AB; Raymond, Shapiro, \& Arnell, 1992), in which the identification of one target negatively affects the report of later-occurring targets, particularly if one or two intervening distractors occur between the two targets.

Some evidence has been provided that there are at least two sources of deficits in sequential search tasksone being the $\mathrm{AB}$ and the other being a task-switching cost if the two successive targets differ in some way (e.g., Allport \& Hsieh, 2001; Chun \& Potter, 2001; Potter, Chun, Banks, \& Muckenhoupt, 1998). Most studies of the $\mathrm{AB}$ have used two different types of targets, such as a white letter embedded in a stream of black letters, followed by a specific black letter probe, such as an X (Raymond et al., 1992). It has been pointed out that different target definitions require different task set configurations (e.g., Allport \& Hsieh, 2001; Rogers \& Monsell, 1995), and the need to reconfigure the search parameters can result in some processing loss until the reconfiguration is complete. Rogers and Monsell have identified both labile and asymptotic costs associated with switching tasks in a simple character classification task. They further demonstrated that such costs can be reduced if the specific task on any trial is precued in some way so that the task set can be configured in advance. However, even simple changes in task requirements can produce costs that are not resolved despite what would appear to be adequate opportunity for reconfiguration (e.g., Arrington \& Logan, 2004; Monsell, Sumner, \& Waters, 2003; Nieuwenhuis \& Monsell, 2002). Task-switching costs are, therefore, a ubiquitous component in many dual-task environments (Arnell, 2001).

It is clear that task set reconfiguration cannot be the only explanation of the $\mathrm{AB}$ phenomenon, since a processing deficit occurs even if the two targets are defined in the same way (Broadbent \& Broadbent, 1987; Juola, Duvuru, \& Peterson, 2000; Potter et al., 1998). Furthermore, the AB effect often shows a characteristic called lag 1 sparing (Potter et al., 1998; Visser, Bischof, \& Di Lollo, 1999), in which T2 is identified more accurately if it immediately follows $\mathrm{T} 1$ than if the lag between the two targets is increased. Any task-switching costs should be strongest at lag 1 and should dissipate over time (Rogers \& Monsell, 1995). Lag 1 sparing presumably results from the opening and closing of an attentional gate (e.g., Sperling \& Weichselgartner, 1995) or from selection for detailed processing of items that meet a target's definition (Chun \& Potter, 1995; Shapiro \& Raymond, 1994). If this gate or selective filter is comparatively sluggish in its response time, relative to the rate of RSVP presentation, both the target and the item following the target can be selected for detailed processing and memory consolidation. When the following item is also a target, it is likely to be processed to a deeper level as well and, thus, be available for later report. However, if T2 occurs after one or two intervening distractors, processes tied up with identifying and consolidating memory for $\mathrm{T} 1$ are often unavailable for $\mathrm{T} 2$, resulting in its loss from memory as it is overwritten by successive items. This type of central-processing bottleneck has been identified as a major source of the AB effect (e.g., Jolicœur, Dell'Acqua, \& Crebolder, 2001).

Visser et al. (1999) have identified potential causes of the lag 1 sparing phenomenon. They examined differences between definitions of $\mathrm{T} 1$ and $\mathrm{T} 2$ in a large corpus of AB studies and found that the second of two successive targets is reported most accurately if the two targets share the same physical and categorical dimensions. If the target-defining features are the same for both targets, this similarity increases the probability that they will be encoded into the same attentional episode. Even singledimensional shifts in target identification tasks produce relatively little loss in identifying the second of two successive targets. However, if the attentional shift between tasks involves several dimensions or the single dimension of location, T2 is identified relatively poorly at short lags, especially at lag 1. Chun and Potter (2001) suggested that the observed amount of lag 1 sparing in an AB task is a sensitive index of task similarity in identifying the two targets (see also, Enns, Visser, Kawahara, \& Di Lollo, 2001).

The present set of experiments was designed to gain insight into causes of processing deficits associated with reporting successive events in a rapidly presented stream of visual items. In the first experiment, either two successive targets were defined in the same way (both targets were either white letters or black digits against a background stream of black letters), or they belonged to different categories (a white letter followed by a black digit, or vice versa within the stream of black letters). Although this single categorical switch should produce some costs relative to the nonswitch conditions, we expected to obtain lag 1 sparing in both conditions (consistent with the conclusions of Visser et al., 1999). It is of some interest to compare trials in which target type is blocked versus randomized, since blocked conditions for target category switch trials might allow for preconfiguration of the attentional set required for target identification, whereas randomized trials would not allow any such preconfiguration. Although the $\mathrm{AB}$ effect has been confounded with task-switching effects in many studies, this effect has been eliminated in others without quantifying the effects of including or eliminating the confounding. Experiment 1 provides one of the first direct comparisons of $\mathrm{AB}$ effects with and without task-switching costs within a single study. Although Kawahara, Zuvic, Enns, and Di Lollo (2003, Experiment 5) recently reported just such a comparison, their manipulation of task switches was between subjects for prepared participants, whereas the task switches in the present Experiment 1 
were unpredictable (in half of the conditions) and were always manipulated within subjects for highly practiced participants. The present design should provide a more powerful basis for evaluating the contribution of task switching to the deficits observable in the AB task.

Experiment 2 included spatial position as an additional variable, with the expectation that lag 1 sparing should not be observed over a shift in position between two successive targets (e.g., Peterson \& Juola, 2000; Visser et al., 1999). Again, it is of interest to compare trials in which target location is blocked versus randomized in order to test for any location expectation or configuration effects. Experiment 2 is apparently the first direct comparison of the relative effects of task- and location-switching within the AB paradigm. Again, both variables were manipulated within subjects for highly practiced participants, and location switches were predictable in half of the conditions and unpredictable in the others. Theoretical explanations of the AB should be able to account for both task- and location-switching effects, in order to determine the relative nature and possible loci of these different causes of temporal-processing deficits in visual attention.

\section{EXPERIMENT 1}

\section{Method}

Participants. The participants were 4 male and 4 female volunteers ranging in age from 21 to 53 years, recruited from among students, faculty, and visitors at the Universidad Autónoma de Madrid. All had normal or corrected-to-normal vision.

Materials. All the stimuli were presented on a Nokia 17-in. color monitor controlled by a desktop computer. They consisted of 8 capital letters and 8 digits making up the set $\{\mathrm{Q}, \mathrm{W}, \mathrm{E}, \mathrm{R}, \mathrm{T}, \mathrm{Y}, \mathrm{U}, \mathrm{P}, 2$, $3,4,5,6,7,8,9\}$. At a viewing distance of about $60 \mathrm{~cm}$, each character subtended $0.5^{\circ}$ or less in horizontal and vertical extension. Each stimulus list consisted of a sequence of 14 individual items preceded and followed by a central fixation asterisk. Each list began and ended with the \# symbol (positions 1 and 14) and included the symbols \% and \& in positions 2 and 3, 2 and 13, or 12 and 13, randomly from trial to trial. The other 10 positions included characters from the critical set, which included 10 letters, 9 letters and $1 \mathrm{nu}-$ meral, or 8 letters and 2 numerals. When a numeral appeared, it was defined as a target - that is, one of the items to be reported. Similarly, on other trials, 0,1 , or 2 letters were defined as targets by showing them in white against a gray screen, as opposed to the black color used for all the numerals and the distractor letters. Each list always included two targets - 2 white letters (LL), a white letter followed by a black numeral (LN), a black numeral followed by a white letter (NL), or two black numerals (NN). On NN trials, all 8 letters in the critical set were used once each as distractors. On the other three types of trials, 1 or 2 of the early letters in the series were repeated later, after all the letters had been presented once, but the target item(s) was never repeated.

The lag between the two targets was varied between zero and five intervening items (called lags 1-6). A set of Latin squares was used to construct 32 different stimulus series at each combination of target type (LL, LN, NL, and NN) and lag (6), to create a total of 768 different trial stimulus lists.

Procedure. Each participant viewed each stimulus list at least two times, once in a blocked target type condition, in which, for four different 192-trial blocks, each of the four target types was held constant, and once in a mixed target type condition, in which all four target types were shown equally often in a random order in four different 192-trial blocks. A session consisted of two blocks, one with target type blocked and one with mixed target types, each preceded by a series of 48 practice trials selected from among the experimental stimuli used in the following block. All the participants completed four sessions, usually run on separate days, made up of eight blocks, for a total of 384 practice trials and 1,536 experimental trials. Thus, all the variables - blocked versus mixed target types (2), target type (4), and lag (6) - were manipulated within subjects, with 32 replications per participant in each cell of the design.

Each session was run in a semidarkened room. The procedure was identical for the two 48-trial practice blocks and the two experimental blocks in each session. One of the blocks in each session was in the blocked target type condition, and in the other one the target types were mixed. The order in which the participants were run in the two block types was counterbalanced both within and between sessions.

Each trial began with the presentation of an asterisk in the middle of the screen. This was a signal for the participant to begin the series, when ready, by pressing the space bar on the computer keyboard. After a 500-msec delay, the asterisk was replaced by the first item in the series (always the symbol \#) and the 10 critical characters embedded between noncharacter symbols. The items were presented for $100 \mathrm{msec}$ each, with no intervening time intervals. T1 was always located in position 3,4 , or 5 , and T2 followed it with a lag of 1-6 (0-5 intervening items). The trial ended with the presentation of a sequential pair of questions (in Spanish, with English translations reported here) appearing in block letters on the screen. The first question was, "What was the name of the first white letter?" on trial type LL, "What was the name of the white letter?" on trial type LN, "What was the name of the black digit?" on trial type NL, and "What was the name of the first black digit?" on trial type NN. Similarly, the second question was "What was the name of the second white letter?" on trial type LL, "What was the name of the black digit?" on trial type LN, "What was the name of the white letter?" on trial type NL, and "What was the name of the second black digit?" on trial type NN. Each question disappeared when one of the critical items was pressed on the keyboard. After the second response, the asterisk reappeared to signal readiness for the next trial.

The participants were instructed to respond on all trials, even if they had to guess, and no feedback was provided. After the last pair of questions in each practice and experimental trial block, instructions appeared that informed the participant about what type of block would appear next or that signaled the end of a session. The participants were told that they could take rest breaks whenever they wanted. Each of the four sessions lasted about 45 min, including two practice blocks and two experimental trial blocks per session (480 total trials per session).

\section{Results}

The relevant data are discussed in two parts. These include the mean numbers of T1s correctly reported (out of 32) for each participant in each cell of the block type (blocked vs. mixed target types) by target type (LL, LN, NL, or NN) by lag (1-6) design. The proportions correct on $\mathrm{T} 2$ items were then conditionalized on correct $\mathrm{T} 1 \mathrm{re-}$ port. These data are discussed in turn.

First-target data. The mean numbers of correctly reported characters for each condition of the design for $\mathrm{T} 1$ only are shown in Figure 1. Here, the data are shown separately for blocked and mixed trial type conditions (see also Table 1). The data were analyzed using a 2 (block type) $\times 4$ (target type) $\times 6$ (lag) within-subjects analysis of variance (ANOVA). In general, digits were re- 


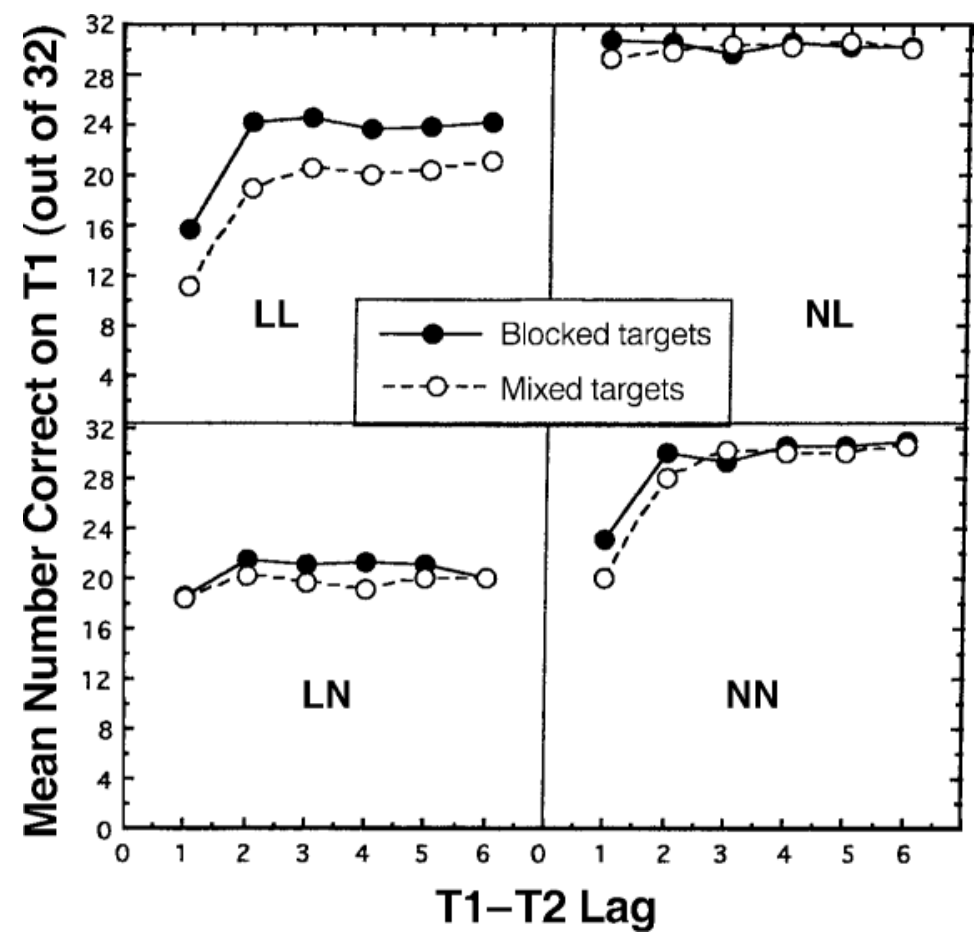

Figure 1. Mean number of correctly reported first targets (T1s) that were letters (left panels) or numbers (right panels), followed by second targets (T2s) that were letters (top panels) or numbers (bottom panels) for blocked versus mixed target types, plotted as a function of T1-T2 lag (Experiment 1).

ported more accurately than letter targets $[92 \%$ vs. $67 \%$; target type main effect, $F(3,21)=12.41, p<.001]$, and report of $\mathrm{T} 1$ was affected only slightly (about $2 \%$ in most conditions) by the blocked/mixed factor, except in the LL condition, in which performance was about $13 \%$ higher on blocked trials $[F(1,7)=19.50, p=.003$, for the main effect, and $F(3,21)=6.92, p=.002$, for the block type $\times$ target type interaction]. Lag also interacted with target type, primarily due to the large difference in accuracy between lags 1 and 2 for the identical target type conditions [LL and NN; $F(5,35)=44.81, p<.001$, for the main effect of lag, and $F(15,105)=10.76, p<$ .001 , for the interaction of lag and target type].

Second-target data. The mean conditionalized proportions of correctly reported characters in each condition for T2 are shown in Figure 2, and the same data, collapsed across lag, are presented in Table 1. These data are the proportions of trials on which T2 was correctly identified, conditionalized on a correct response for T1 (T2|T1) for blocked and mixed trial type conditions. The data were analyzed using a 2 (T1 type: $\mathrm{L}$ or $\mathrm{N}) \times 2(\mathrm{~T} 2$ type $) \times 2$ (blocked vs. mixed trial types) $\times 6$ (lag) ANOVA. In general, the conditionalized report of T2 was affected only slightly by the blocked/mixed factor. When T1 was a letter, T2 was reported about $10 \%$ more accurately in the blocked condition, but the blocked advantage was only about $3 \%$ higher when a digit occurred first $[F(1,7)=$ $32.59, p=.001$, for the main effect, and $F(1,7)=7.84$, $p=.027$, for the block type $\times \mathrm{T} 1$ type interaction].

The lag effect was significant $[F(5,35)=19.61, p<$ $.001]$, reflecting the U-shaped functions in the conditionalized $\mathrm{T} 2$ report data. When $\mathrm{T} 1$ was correctly re-

Table 1

Mean Percentages of First Targets (T1s) and Conditionalized Second Targets (T2s) Reported in Each Condition in Experiment 1, Collapsed Across Lag

\begin{tabular}{|c|c|c|c|c|c|c|c|c|}
\hline & \multicolumn{8}{|c|}{ Condition } \\
\hline & \multicolumn{2}{|c|}{ LL } & \multicolumn{2}{|c|}{$\mathrm{LN}$} & \multicolumn{2}{|c|}{ NL } & \multicolumn{2}{|c|}{$\mathrm{NN}$} \\
\hline & Blocked & Mixed & Blocked & Mixed & Blocked & Mixed & Blocked & Mixed \\
\hline $\mathrm{T} 1$ & 71.2 & 58.5 & 64.3 & 61.0 & 95.5 & 94.3 & 90.3 & 87.8 \\
\hline $\mathrm{T} 2 \mid \mathrm{T} 1$ & 71.0 & 60.5 & 86.8 & 76.5 & 57.3 & 55.3 & 92.7 & 88.8 \\
\hline
\end{tabular}

Note-L, letter; N, number. 


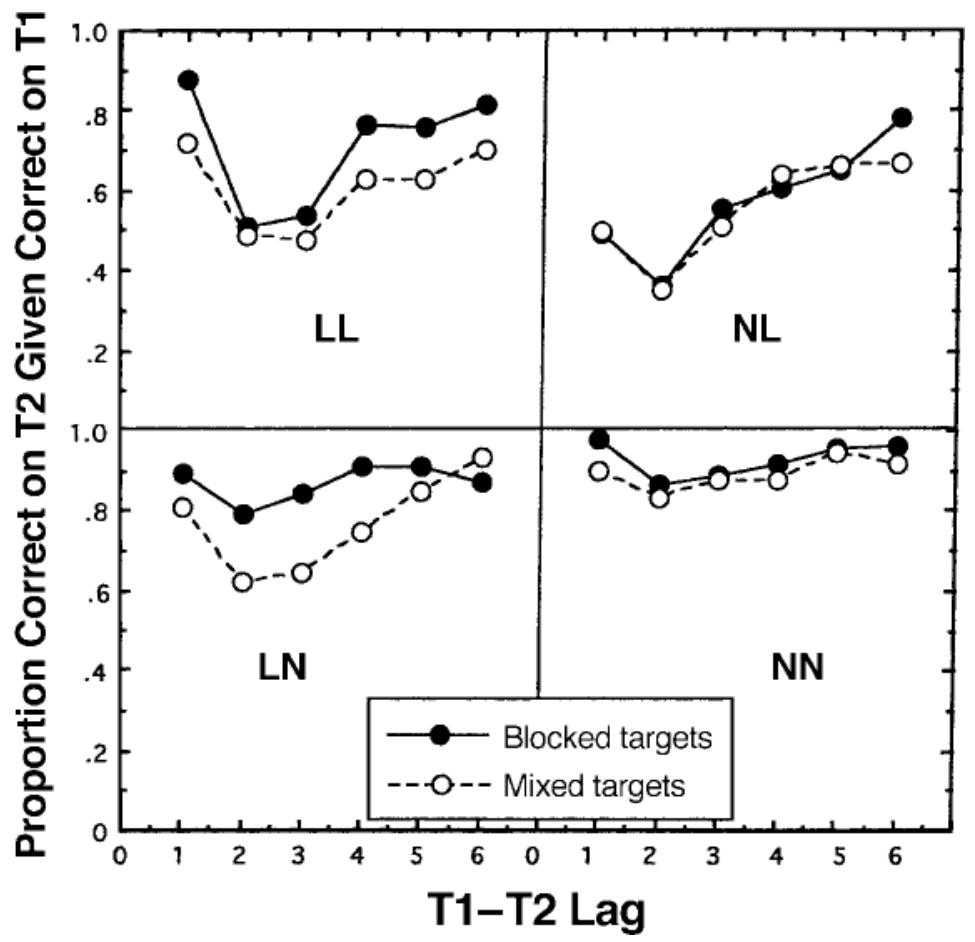

Figure 2. Mean proportions of correct second-target (T2) responses conditionalized on correct first-target (T1) responses for T2s that were letters (top panels) or numbers (bottom panels) following T1s that were letters (left panels) or numbers (right panels) for blocked versus mixed target types plotted as a function of T1-T2 lag (Experiment 1).

ported, report of $\mathrm{T} 2$ was usually correct as well, if there were no intervening items between $\mathrm{T} 1$ and $\mathrm{T} 2$ (lag 1$)$. As lag increased, performance fell in all conditions, reaching the lowest levels at lag 2 or 3 before returning to about the level of performance at lag 1 by lags $4-6$. This is the lag 1 sparing phenomenon commonly found in the $\mathrm{AB}$ literature, at least for tasks involving no, or only a simple, switch between tasks defining T1 and T2 (e.g., Visser et al., 1999).

Performance was higher overall for $\mathrm{T} 2$ if it was a digit $(86 \%)$ than if it was a letter $(61 \%)[F(1,7)=14.97, p=$ $.006]$. However, all of the main effects entered into a significant blocked/mixed $\times$ T1 type $\times$ T2 type $\times$ lag interaction $[F(5,35)=3.56, p=.01]$. This interaction was probably due to the relatively flat lag effects in the LN condition for blocked targets and in the NN condition for blocked and mixed targets, because of ceiling effects in these conditions, in which $\mathrm{T} 2$ was a digit.

Finally, the T2|T1 data show significant switching costs due to a change in target category. When the data are collapsed across lag, performance was lower for digit targets that were preceded by letters (LN trials, $81.6 \%$ ) than for digits preceded by digits (NN trials, 90.8\%). Similarly, letter targets were reported more accurately if they were preceded by letters (LL trials, 65.8\%) than if they were preceded by digits [NL trials, 56.3\%; T1 type $\times$ T2 type interaction, $F(1,7)=54.47, p<.001]$. The cost of switching from one target category to the other was slightly greater than $9 \%$ overall for both types of targets, indicating symmetrical costs of switching between digits and letters. When the data were combined across target types, all the participants showed a target category switch cost of between $4 \%$ and $14 \%[t(7)=6.78, p<$ $.001]$. One would expect that if the difference in results between same character targets (NN and LL trials) and different character trials (NL and LN trials) were actually due to the costs of switching target category, these switching costs should be larger at short lags (Rogers \& Monsell, 1995, Experiment 3). Indeed the mean T2|T1 differences between same character targets and different character targets was $20 \%$ at lag $1,14 \%$ at lag 2 , and about 5\% at lags 3-6 (see Figure 3).

Somewhat surprisingly, there were no differences in target category switch costs between the mixed and the blocked target type conditions. It might have been expected that a predictable category switch would have led to strategic preconfigurations of the target filters, resulting in an immediate or delayed reduction of task-switching costs in the blocked target type condition (e.g., Arrington \& Logan, 2004; Meiran, 2000). However, the overall 


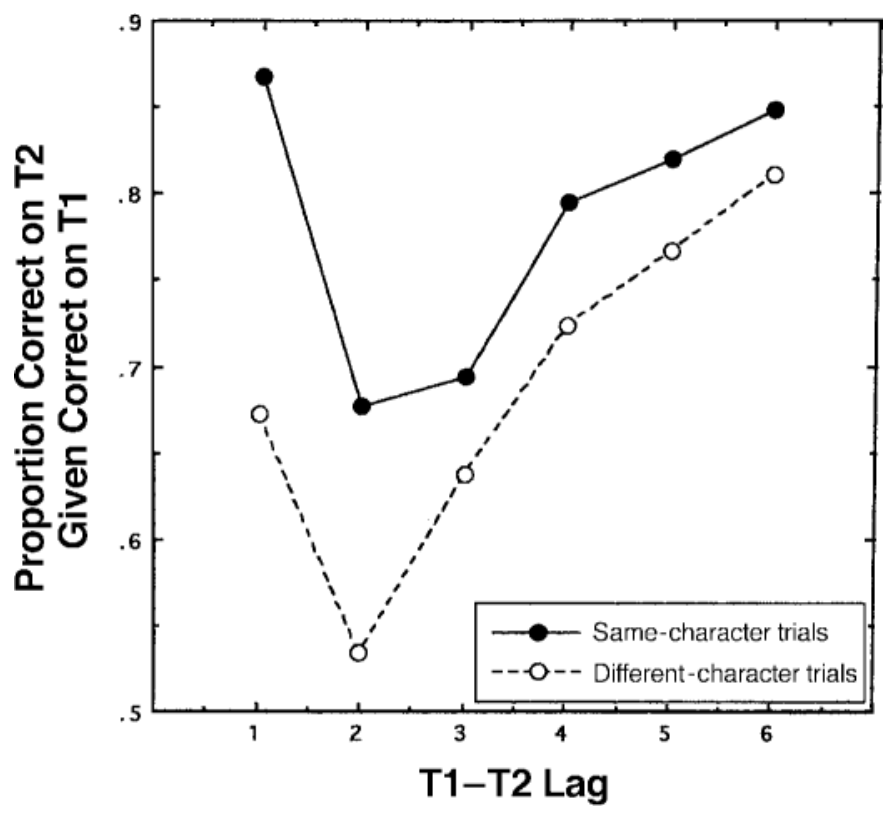

Figure 3. Mean proportions of correct second-target (T2) responses conditionalized on correct first-target (T1) responses for targets from the same category (LL and NN trials) versus targets from different categories (LN and NL trials) plotted as a function of T1-T2 lag (Experiment 1).

switch costs were about $9 \%$ in both the blocked and the mixed trial type conditions, and there were no differences in accuracy rates between conditions across lag.

\section{Discussion}

The T2|T1 data replicate many similar experiments in showing a U-shaped AB function (see Visser et al., 1999, for a review). That is, T2 was well reported if it occurred at lag 1 , and performance dropped off at lag 2 and, sometimes, lag 3 , only to recover to about the level of lag 1 at the longer lags. Thus, the data showed the lag 1 sparing phenomenon, although the magnitude of lag 1 sparing was less for trials with target category switches (letter followed by digit, or vice versa) than for trials with two targets from the same category (both letters or both numerals; see Figure 3). Thus, the cost of a category shift reduced performance on $\mathrm{T} 2$ report, especially at short T1-T2 lags, but the general characteristics of the AB effect remain.

In Visser et al.'s (1999) terms, a simple shift in target category might delay $\mathrm{T} 2$ processing, but it is generally insufficient to prevent a successive $\mathrm{T} 1-\mathrm{T} 2$ pair from being incorporated into the same attentional episode and being encoded together to a deeper level that allows memory consolidation and retention for later report. Longer lags reduce, but do not eliminate, task-switching effects, replicating both the transient and the asymptotic nature of the task-switching costs found by Rogers and Monsell (1995). In Kawahara et al.'s (2003) model, task shifts are proposed to act much the same as increases in T1- processing difficulty (e.g., Visser \& Bischof, 2000). That is, the task-shifting cost adds a delay in addition to the delay caused by $\mathrm{T} 1$ processing, and while $\mathrm{T} 2$ processing is thus delayed, it is more vulnerable to masking from subsequent items.

Blocking target types had a consistent effect on the conditional report of T2 when T1 was a letter, but the effect was smaller and inconsistent when $\mathrm{T} 1$ was a numeral. Apparently, because numeral targets were more easily detected than letter targets, preparation for a digit yielded little gain in $\mathrm{T} 1$ processing when the target type was known in advance. Preparation for a letter, however, facilitated $\mathrm{T} 1$ processing and resulted in higher levels of T2 performance, since resources could then be more quickly released from dealing with T1. Seiffert and Di Lollo (1997) reported a similar effect of T1-processing difficulty on the size of the AB. The advantage for numeral over letter targets in Experiment 1 could be due to any of several factors, including greater likelihood of confusion of letter targets with the distractor letters and differences in targetdefining and response features for the two types of targets. Similar results were found in the following experiments, so we will postpone a more thorough analysis of target type effects to the General Discussion section.

The T1 report data replicate similar findings by Broadbent and Broadbent (1987), who found that report accuracy for $\mathrm{T} 1$ was lower if $\mathrm{T} 2$ followed immediately than if there were one or more intervening items between $\mathrm{T} 1$ and T2. They attributed this effect to a type of horserace between recognition processes for successive targets, in 
which the first one processed tends to suppress processing of the other. Thus, a low T1 report at lag 1 could be due to interference between similar recognition processes, especially when the two targets are of the same type (LL or NN). Alternatively, it could be the case (as Weichselgartner \& Sperling, 1987, have argued) that both targets are recognized but order information is often lost, so that sometimes they are reported in the correct order and sometimes their order is reversed (see also Chun \& Potter, 1995, for a similar result). Unfortunately, responses were coded only as being correct or incorrect in Experiment 1, so such a post hoc analysis of the data could not be made. Experiment 1A was designed, therefore, to collect evidence for these two explanations for the relatively poor performance for $\mathrm{T} 1$ at lag 1 in the $\mathrm{LL}$ and $\mathrm{NN}$ conditions by replicating parts of Experiment 1 while recording the responses made on each trial. These could then be compared with the stimulus list for that trial, in order to determine whether errors were reversals or intrusions.

\section{EXPERIMENT 1A}

Experiment 1A was a partial replication of Experiment 1 that was designed primarily to determine whether order reversal errors could have been a major cause of reduced accuracy for T1 reports at short lags in Experiment 1.

\section{Method}

Seven of the 8 participants from Experiment 1 participated in Experiment $1 \mathrm{~A}$, and 1 new (male) participant was selected from the same pool. (The participant with the lowest overall accuracy rate in Experiment 1 was replaced, having achieved $63 \%$ correct on $\mathrm{T} 2 \mid \mathrm{T} 1$, as opposed to over $75 \%$ for the other 7 participants.) The conditions were exactly the same, except that only LL and NN trials in the blocked target type condition were run and the participants viewed 192 trials in each condition, plus practice trials, in each of two separate sessions.

\section{Results}

Table 2 shows the data for all conditions run in Experiment $1 \mathrm{~A}$. The first row for the T1 report data are uncorrected and show a pattern similar to that observed in comparable conditions in Experiment 1. The 7\% improvement in overall performance, relative to Experiment 1, was probably due to a practice effect plus the replace- ment of the least accurate participant in Experiment 1, and it was largely confined to the LL condition, since the data in the NN condition are closer to ceiling. The data show the same relative deficits in reporting $\mathrm{T} 1$ in the lag 1 condition and reporting T2|T1 in the lag 2 condition on NN trials and at lags 2 and 3 for LL trials as those found in Experiment 1. However, a relatively large proportion of these errors could be identified as reversals; that is, both targets were correctly reported, but in the wrong order. When the data were corrected for reversal errors, as is shown in the rows marked with an * in Table 2, a somewhat different picture emerged.

Of the 256 trials overall at lag 1 in condition LL, 54 of them resulted in errors on both $\mathrm{T} 1$ and $\mathrm{T} 2$, and 19 of these $(35 \%)$ were due to reversal errors. Similarly, in the NN condition, the participants made 67 errors on both $\mathrm{T} 1$ and $\mathrm{T} 2$, but 57 of these (85\%) were reversal errors. By treating reversal errors as correct responses, the difference between error rates at lags 1 and 2 diminished for the T1 report data in the LL condition, from $23 \%$ in the raw data to $16 \%$ in the corrected data, but this difference remained significant $[t(7)=4.30, p<.001]$. In the $\mathrm{NN}$ condition, the lag 1-lag 2 difference in T1 error rates diminished from $22 \%$ in the raw data to $5 \%$ in the corrected data, and the latter difference was not significantly different from zero $[t(7)=1.06, p>.05]$.

In the conditionalized $\mathrm{T} 2$ report data, the correction for reversal errors produced little other than a slight increase in performance at the shorter lags. In the LL condition, the difference between percentages of correct responses at lags 1 and 2 were $22.8 \%$ for the raw data and $22.1 \%$ for the corrected data, and these respective differences were $10.3 \%$ and $10.2 \%$ for the NN condition. There were no important changes in either $\mathrm{T} 1$ or conditionalized $\mathrm{T} 2$ report data due to correction for reversal errors after lag 2.

\section{Discussion}

A significant number of reversal errors explains almost all of the lag 1 deficit data for NN trials, but not for LL trials. That is, besides being easier to recognize and report, two successive digits seem to produce less processing interference than do two successive letter targets. Even when corrected for reversal errors, there remains a significant deficit for reporting the first of two target let-

Table 2

Mean Percentages of First Targets (T1s) and Conditionalized Second Targets (T2s) Reported in Each Condition in Experiment 1A

Condition

\begin{tabular}{|c|c|c|c|c|c|c|c|c|c|c|c|c|}
\hline & \multicolumn{12}{|c|}{ Condition } \\
\hline & \multicolumn{6}{|c|}{ LL } & \multicolumn{6}{|c|}{$\mathrm{NN}$} \\
\hline & $\overline{L a g} 1$ & Lag 2 & Lag 3 & Lag 4 & Lag 5 & $\operatorname{Lag} 6$ & Lag 1 & $\operatorname{Lag} 2$ & Lag 3 & Lag 4 & Lag 5 & Lag 6 \\
\hline $\mathrm{T} 1$ & 63.3 & 86.7 & 88.7 & 89.1 & 85.9 & 87.9 & 71.1 & 93.4 & 93.0 & 97.3 & 95.3 & 94.9 \\
\hline $\mathrm{T} 1 *$ & 70.7 & 87.1 & 88.7 & 89.1 & 85.9 & 87.9 & 93.4 & 98.0 & 94.1 & 97.7 & 95.7 & 94.9 \\
\hline $\mathrm{T} 2 \mid \mathrm{T} 1$ & 86.8 & 64.0 & 71.6 & 85.2 & 87.2 & 93.0 & 97.9 & 87.6 & 95.2 & 95.2 & 94.9 & 96.6 \\
\hline $\mathrm{T} 2 \mid \mathrm{T} 1 *$ & 90.0 & 67.9 & 71.6 & 85.2 & 87.2 & 93.0 & 98.4 & 88.2 & 95.3 & 95.2 & 94.9 & 96.6 \\
\hline
\end{tabular}

Note-The first row in each condition shows the raw data, and the second row $(*)$ shows the data corrected by including reversal errors as correct responses. L, letter; $\mathrm{N}$, number. 
ters when they are successive, as opposed to when there are one or more intervening distractor letters. This deficit is probably due to some inhibitory mechanism, such as that suggested by Broadbent and Broadbent (1987), in which competing letter responses produce interference so that the first one processed to the level of report can suppress report of the second one. Although similar processes might be at work for numeral targets, the lag 1 deficit corrected for reversal errors is not significant, and the greater ease of recognizing and reporting digit targets in the present task apparently relieves responses from much of the destructive interference affecting letter targets. Undoubtedly, some of this interference is due to the fact that all the distractors were letters. Other researchers have shown that target report accuracy in the $\mathrm{AB}$ paradigm decreases with the relative confusability of the target and the distractor items, an effect that would favor report of the numeral targets in the present experiments (Chun \& Potter, 1995; Isaak, Shapiro, \& Martin, 1999).

\section{EXPERIMENT 2}

The original AB research (Raymond et al., 1992) used two different types of items for T1 (to be identified) and the following probe (to be detected). Therefore, it is likely that both a task-switching cost and a loss due to $\mathrm{AB}$ affected detection of T2 (probe item). Eliminating a task switch removes this cost but leaves the $\mathrm{AB}$ effect largely intact (Arnell, 2001). Location switches between the two items to be reported produce a different result, however. If the two targets are located at different positions, the lag 1 sparing phenomenon generally is greatly reduced (Shih, 2000) or disappears completely (Peterson \& Juola, 2000), although performance at later lags shows a processing deficit followed by recovery that is consistent with similar conditions in the typical AB task. In Experiment 2, task switches and location switches were combined in some conditions in order to determine whether the different effects of the two types of switches extend beyond the lag 1 sparing phenomenon to more general aspects of the $\mathrm{AB}$.

\section{Method}

Participants. The participants were 8 volunteers recruited from among students, faculty, and visitors at the Universidad Autónoma de Madrid. They were 6 males and 2 females ranging in age from 21 to 53 years. All had normal or corrected-to-normal vision, and 6 of the 8 had participated previously in Experiments 1 and/or 1A.

Materials. The stimulus materials and apparatus were the same as in the previous experiments. The only difference was in the position of the character streams. In Experiment 2, a pair of streams was presented, on either side of a fixation point $(+$ sign $)$. The viewing distance was not precisely controlled, but from a typical sitting position, the two streams were between $1^{\circ}$ and $2^{\circ}$ of visual angle apart (the center-to-center distance between characters was about $1.6^{\circ}$ from a typical viewing distance of $60 \mathrm{~cm}$ ). Each stream was identical to the ones shown in Experiment 1, but care was taken to make sure that no distractor letter was repeated within two successive positions on either side, and target items were never repeated within a trial. As in Experiment 1, the T1-T2 lag was varied between zero and five intervening items, either within the same stream or between the two streams. Only the mixed trial type condition from Experiment 1 was run (to simplify the design and to avoid some problems of ceiling effects observed in Experiment 1), so all target type pairs (LL, LN, NL, and NN) occurred randomly, but with equal probability, within sessions. The locations of the targets were controlled in two conditions, however. In mixed location blocks, the target could occur on either side, so that left-left, left-right, right-left, and right-right target locations were equally likely. In the blocked location conditions, only the left-right or the right-left target location pairings were used in separate blocks, so the participants always knew on which side to expect T1 and they knew to expect T2 on the opposite side. The left-left and right-right conditions were not included in the blocked location trials, since this would have reduced the conditions to a replication of Experiment 1, with an irrelevant stream to one side. The combinations of target type (4) and lag (6) were replicated 32 times with different stimulus lists to create a total of 768 different trial stimuli.

Procedure. Each participant viewed each stimulus no more than once in the experimental trials, and some of them were randomly selected for use on practice trials. Different halves of the stimuli were selected for each participant for the blocked and the mixed location conditions. In the blocked location condition, target type (LL, LN, NL, and NN) was determined randomly for each trial for two 192-trial blocks, but the target locations were held constant. In one of these blocks, T1 was always on the left side, and T2 was always on the right side. These sides were reversed in the other block in the blocked location condition. In the mixed location condition, all the stimuli and all four target types were shown equally often in a random order in four different 192-trial blocks. In each of these four blocks, the locations of T1 and T2 were independent, so that they could both appear on the same side (left or right) or each could appear on either side, in either order. The experiment always began with a mixed location block, followed by one of the blocked location blocks, then two mixed location blocks, the other blocked location block, and finally the last mixed location block. These six blocks consisted of 48 practice trials, with materials randomly selected from the experimental stimulus lists, followed by 192 experimental trials. All other details of the procedure were the same as those in Experiments 1 and $1 \mathrm{~A}$.

\section{Results}

The relevant data will be discussed in two parts. First, the mean proportions of correct reports of $\mathrm{T} 1$ were found for each participant in each cell of the block type (blocked location vs. mixed location $) \times \mathrm{T} 1$ type $(\mathrm{L}$ or $\mathrm{N}) \times$ location (left or right) $\times$ lag (1-6) design. The proportions correct on the T2 item were then conditionalized on correct $\mathrm{T} 1$ report. These data will be discussed in turn.

First-target data. The mean proportions of correctly reported characters for each condition of the design for T1 only are shown in Figure 4. Here, the data are presented separately for the blocked and the mixed location conditions for letter and digit targets presented on either side of the fixation point. In general, digits $(92 \%)$ were reported more accurately than letter targets $[79 \%$; target type main effect, $F(1,7)=10.08, p=.016]$, left-side targets were reported more accurately than those on the right $[88 \%$ vs. $82 \%$; location main effect, $F(1,7)=12.26, p=$ $.010]$, and the blocked location condition resulted in a higher proportion of correct responses than the mixed location condition $[90 \%$ vs. $80 \%$; blocking main effect, $F(1,7)=62.70, p<.001]$. The lag effect was also sig- 


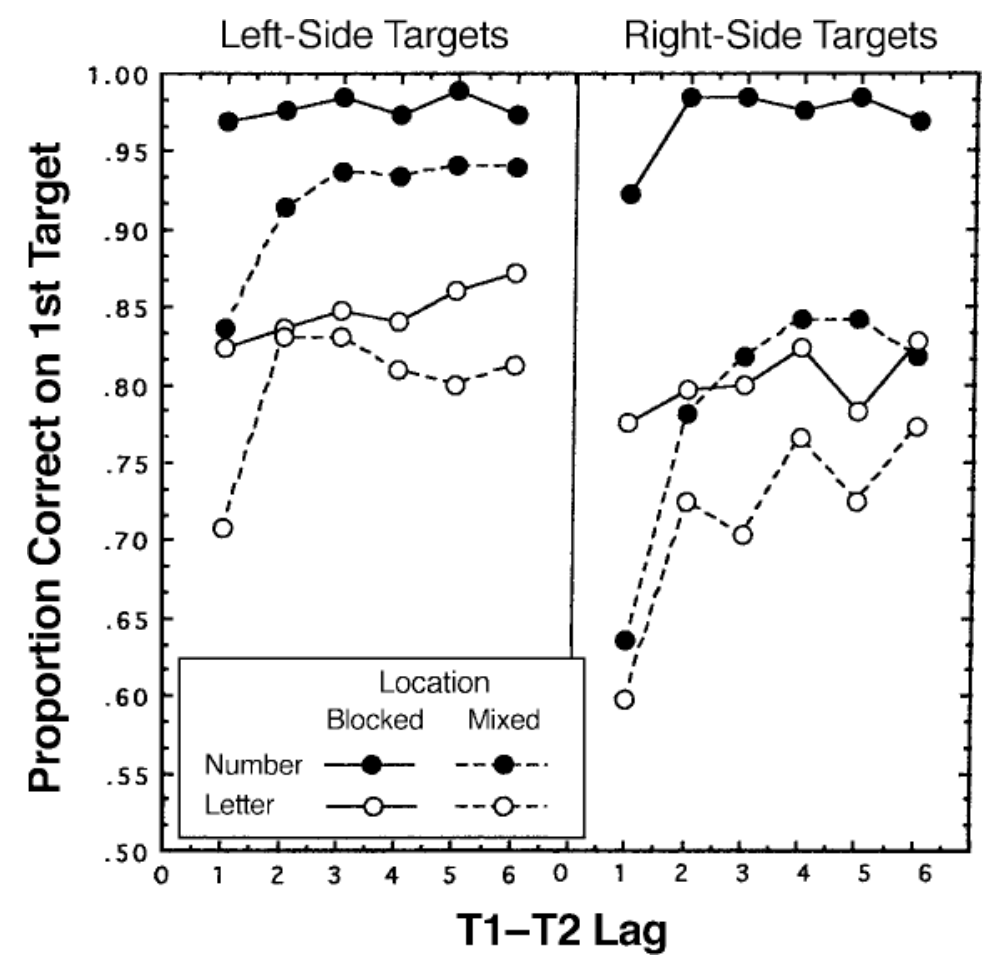

Figure 4. Mean number of correctly reported first targets that were letters or numbers presented in the left or the right rapid serial visual presentation stream for conditions in which the position of the first target (T1) was blocked versus randomly located to the left or to the right (Experiment 2).

nificant $[F(5,35)=6.26, p<.001]$. Most of the lag effect was due to the poor performance at lag 1 . Furthermore, the lag 1 deficit for $\mathrm{T} 1$ report was greatest for $\mathrm{LL}$ and $\mathrm{NN}$ trials in the mixed location condition when the two targets were presented on the same side of the fixation point (see Table 3 ).

Significant interactions included target type $\times$ blocked/ mixed location $\times$ target location and target type $\times$ blocked $/$ mixed location $\times$ lag $[F(1,7)=13.26, p=$ .008 , and $F(5,35)=4.57, p=.003$, respectively].

As in Experiment 1, the percentage of correct T1 reports increased less from lag 1 to lag 2 for mixed target types [LN and NL trials, $P(\mathrm{~T} 1$ correct $)=.81$ and .86 for lags 1 and 2, respectively] than for same target type trials [LL and NN trials, $P$ (T1 correct) $=.69$ and .82 , for lags 1 and 2, respectively]. The lag data are presented for particular conditions in Table 3. Here, the difference in T1 reports for lags 1 and 2 can be compared for LL and $\mathrm{NN}$ trials in the blocked and the mixed location conditions for trials with both targets on the left or on the right (top half of Table 3 ) or when both targets were presented to the same side or to different sides (bottom half of Table 3).

The data in Table 3 clearly show that the largest difference in T1 report accuracy between lag 1 and lag 2 occurred when LL and NN targets were presented to the same side of the display. These are exactly the conditions in which it would be most likely that the order of two targets that had been correctly identified would be confused. Therefore, as in Experiment 1A, an analysis was made of the data corrected for order reversal errors in reporting T1 and T2 in Experiment 2. When reversal errors were counted as correct responses, the large deficits found in T1 report at lag 1 largely, but not completely, disappeared. The corrected data are shown in Table 4. The lag effect remains significant $[F(5,35)=2.78, p=$ .032], but now it enters into no significant interactions. The interaction that remains, however, shows that the participants clearly were biased to attend to the left stream in the mixed location condition. That is, when T1 occurred on the left, overall performance was $91 \%$ correct in the blocked location condition and $88 \%$ in the mixed location condition. When T1 occurred on the right, the blocked and the mixed location conditions showed mean correct responses of $89 \%$ and $76 \%$, respectively. In other words, performance was about as good (3\% difference) for left-side targets as for right-side ones when the location of T1 was known. However, when T1 could occur on either side with equal probability, there was a $13 \%$ advantage for left-side targets.

Second-target data. The mean proportions of correctly reported characters in each condition of the design 
Table 3

Mean Percentage of T1 Targets Correctly Reported for Lags of 1 and 2 Between T1 and T2 for Selected Conditions of Experiment 2

\begin{tabular}{|c|c|c|c|c|}
\hline \multirow[b]{3}{*}{ Location } & \multicolumn{4}{|c|}{ Condition } \\
\hline & \multicolumn{2}{|c|}{ LL Trials, T1 Left Side } & \multicolumn{2}{|c|}{ LL Trials, T1 Right Side } \\
\hline & Lag 1 & Lag 2 & Lag 1 & $\operatorname{Lag} 2$ \\
\hline Blocked & 82.0 & 78.9 & 80.5 & 78.9 \\
\hline \multirow[t]{3}{*}{ Mixed } & 62.6 & 84.0 & 51.6 & 69.5 \\
\hline & \multicolumn{2}{|c|}{ NN Trials, T1 Left Side } & \multicolumn{2}{|c|}{ NN Trials, T1 Right Side } \\
\hline & Lag 1 & Lag 2 & Lag 1 & Lag 2 \\
\hline Blocked & 97.7 & 99.2 & 86.7 & 98.4 \\
\hline \multirow[t]{3}{*}{ Mixed } & 73.8 & 87.9 & 52.7 & 71.9 \\
\hline & \multicolumn{2}{|c|}{ LL Trials, Same Side } & \multicolumn{2}{|c|}{ LL Trials, Different Sides } \\
\hline & Lag 1 & Lag 2 & Lag 1 & Lag 2 \\
\hline Blocked & - & - & 81.3 & 78.9 \\
\hline \multirow[t]{3}{*}{ Mixed } & 38.3 & 74.2 & 75.8 & 79.3 \\
\hline & \multicolumn{2}{|c|}{ NN Trials, Same Side } & \multicolumn{2}{|c|}{ NN Trials, Different Sides } \\
\hline & Lag 1 & Lag 2 & Lag 1 & Lag 2 \\
\hline Blocked & - & - & 92.2 & 98.8 \\
\hline Mixed & 54.7 & 80.5 & 71.9 & 79.3 \\
\hline
\end{tabular}

for T2 only are shown in Figure 5. The figure plots the proportions of trials on which $\mathrm{T} 2$ was correctly identified, conditionalized on a correct response for T1 (T2|T1) for blocked and mixed location conditions, and within the mixed location condition, whether the two targets appeared on the same or different sides of the fixation point. The data were analyzed using a T1 target type $\times$ T2 target type $\times$ location condition (blocked same sides, mixed same sides, and mixed different sides) $\times$ lag (1-6) ANOVA. One of the main results was a lag $\times$ location condition interaction $[F(10,70)=9.35, p<.001]$. In general, targets presented to the same side resulted in lag 1 sparing, whereas lag 1 sparing was eliminated in the different-side conditions, even when the sides were blocked and the participants knew on which sides the two targets would appear. Despite lag 1 sparing, the lag functions also appear to be flatter in the same-side condition than in the other two conditions, with the data generally reflecting higher levels of report accuracy at short lags in the mixed location, same-side condition, but accuracy becomes lower than that in the two different-side conditions at longer lags.
A second main result is the interaction of $\mathrm{T} 1$ target type with $\mathrm{T} 2$ target type, showing an advantage for NN and LL trials over NL and LN trials [the task-switching cost; $F(1,7)=23.84, p=.002]$. T1 and T2 types also interacted with lag $[F(5,35)=6.32, p<.001]$, indicating that, as in Experiment 1, the task-switching cost decreased with lag. The mean T2|T1 differences between same-character targets and different-character targets were $11 \%$ at lag $1,10 \%$ at lag 2 , and about $2 \%$ at lags 3-6.

Finally, a significant main effect for location $[F(2,14)=$ $12.7, p=.001]$ showed that performance collapsed across lags was highest in the blocked location condition ( $81 \%$ correct on T2|T1), as compared with mean report accuracies of $77 \%$ in the mixed location, same-side condition and $74 \%$ in the mixed location, different-side condition. The effects of $\mathrm{T} 1$ and $\mathrm{T} 2$ type on $\mathrm{T} 2$ report accuracy $[F(1,7)=4.00, p=.086$, and $F(1,7)=10.53, p=.014$, respectively] reflect the overall levels of performance for the four target types (LL, 73\%; NL, 69\%; LN, 82\%; NN, $88 \%)$. These data demonstrate an advantage for digits (14\%) and the cost of task switching (5\%).

As in Experiment 1, the T2|T1 data show significant switching costs due to a change in target category. In Experiment 2, we first corrected the data for reversal errors to provide a more sensitive test for switching costs, since the data for nonswitched targets (NN and LL trials) were artificially lowered by reversal errors (although the correction factor resulted in a much smaller difference in the $\mathrm{T} 2$ data than in the T1 results). A final analysis was performed on the data corrected for reversal errors, in order to test for the effects of location switching and task switching. The data were classified into a lag $(6) \times 10-$ cation (3) $\times$ T2 type (2) $\times$ task switch (2) ANOVA design. Although the effects of both location $[F(2,14)=$ $12.8, p<.001]$ and task switch versus no task switch $[F(1,7)=23.8, p=.002]$ were significant, they did not enter into any significant interactions with each other, suggesting that task and location switching have additive effects on attentional processes. In order to confirm this apparent additivity of costs, we examined the data at lag 1 (when both task-switching and location-switching costs were at their maximum) in the mixed location conditions only, since in these trials, there was complete uncertainty about both task and location for $\mathrm{T} 2$. We found

Table 4

Mean Percentages of First Targets Correctly Reported (Corrected for Reversal Errors) for the (Uncorrected) Data Shown in Figure 4 (Experiment 2)

\begin{tabular}{|c|c|c|c|c|c|c|c|c|c|c|c|c|}
\hline & \multicolumn{6}{|c|}{ Left Side } & \multicolumn{6}{|c|}{ Right Side } \\
\hline & Lag 1 & Lag 2 & Lag 3 & Lag 4 & Lag 5 & Lag 6 & Lag 1 & Lag 2 & Lag 3 & Lag 4 & Lag 5 & $\operatorname{Lag} 6$ \\
\hline \multicolumn{13}{|c|}{ Numbers } \\
\hline Blocked location & 97 & 98 & 99 & 97 & 99 & 98 & 93 & 99 & 99 & 98 & 98 & 97 \\
\hline Mixed location & 95 & 96 & 95 & 96 & 94 & 93 & 75 & 78 & 82 & 84 & 84 & 82 \\
\hline \multicolumn{13}{|c|}{ Letters } \\
\hline Blocked location & 82 & 84 & 85 & 84 & 86 & 87 & 78 & 80 & 80 & 82 & 78 & 83 \\
\hline Mixed location & 82 & 84 & 84 & 80 & 80 & 81 & 73 & 76 & 70 & 77 & 72 & 77 \\
\hline
\end{tabular}




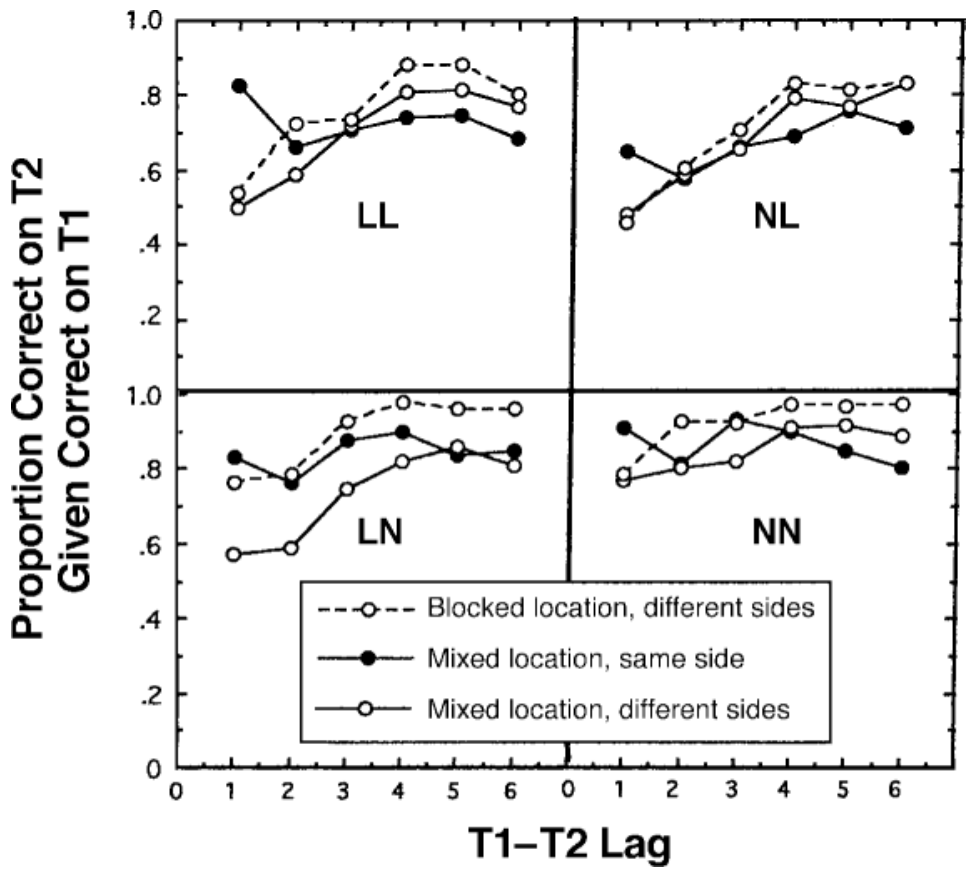

Figure 5. Mean proportions of correct second-target (T2) responses conditionalized on correct first-target (T1) responses for T2s that were letters (top panels) or numbers (bottom panels) following T1s that were letters (left panels) or numbers (right panels) for conditions in which $\mathrm{T} 1$ and $\mathrm{T} 2$ positions were blocked versus conditions in which they were mixed and occurred on either the same side or different sides, plotted as a function of T1-T2 lag (Experiment 2).

that the mean task-switching cost was $14 \%$, the mean location-switching cost was $23 \%$, and the cost of switching both task and location was $37 \%$.

\section{Discussion}

The T1 data largely replicated the results of Experiments 1 and $1 \mathrm{~A}$, except that reversal errors were less common if the two targets occurred on opposite sides of the fixation point, as would be expected. The T2 data showed similar task-switching costs as in Experiment 1, and when the data were corrected for reversal errors, they simply added to the costs of a location switch between $\mathrm{T} 1$ and T2. Location switching by itself eliminated the lag 1 sparing phenomenon, as would be expected on the basis of Visser et al.'s (1999) review of the AB literature. Furthermore, the AB effect was largely the same on both sides of the fixation point after the first lag, indicating that location-switching effects were largely limited to lag 1 (a result also found by Peterson \& Juola, 2000).

It is interesting to note that the elimination of lag 1 sparing was found even when the participants knew on which side of the display each target would appear. Apparently, preparation for location was as ineffective as preparation for target type in dealing with switching costs. It could well be that the attentional cost necessary for preparing a reconfiguration of the set for target type or location in the present tasks negated any gain likely to be had from a successful preparation. Nieuwenhuis and Monsell (2002) have reported other instances in which participants were apparently unable to prepare themselves in a relatively short time to avoid the costs of a simple task switch.

The lag effects showed another interesting trend in the comparison of same- and different-side targets in the mixed location condition. When the participants had no idea about the locations of $\mathrm{T} 1$ and $\mathrm{T} 2$, the conditional report of T2 was higher if it occurred on the same side as T1 for short lags (1-3). However, for longer lags (4-6), performance was actually better if the two targets appeared on opposite sides. This result is reminiscent of the inhibition-of-return phenomenon (Posner \& Cohen, 1984), in which an uninformative peripheral cue leads to an immediate advantage in processing a target at its location. However, after a delay of a few hundred milliseconds, performance decreased and was replaced by an advantage at other possible target locations. Even if attention was distributed fairly evenly over the two streams in the mixed location condition, the appearance of a target in one of the streams seems to have drawn attention to that stream, resulting in a same-stream advantage at short lags. At longer lags, however, just as in the inhibition-ofreturn phenomenon, attention seems to have "rebounded" away from a focus on T1's location to produce an advan- 
tage for the other stream. This effect is unlike the traditional inhibition-of-return result, in that it is triggered by an endogenous cue (the meaning of a color or category change). Yet with highly practiced participants and a small and well-known set of targets, the shift in attention first toward a target and then away from its location mimics the time course of inhibition of return, using exogenous cues.

\section{GENERAL DISCUSSION}

The search for two distinct target characters among a homogeneous set of distractors in an RSVP stream was shown to be affected by at least four variables: (1) the distinctive features that defined the targets and differentiated them from the distractors, (2) the lag between the two targets, (3) whether the targets belonged to the same category (letters or digits) or were switched between categories, and (4) whether the two targets occurred in the same location or were displaced horizontally by less than $2^{\circ}$. In addition, prior knowledge was sometimes provided about Variable 1, 3, or 4 above, but lag was always varied unpredictably. The effects of these variables, and their interactions, will be discussed in turn, before we consider how their combined effects influence item recognition and retention in a sequential visual search task.

\section{Target Definition}

In all the experiments reported here, black digits were reported more accurately than white letters within a stream of black letter distractors. There are several possible explanations for the advantage for numerals. First, for digits, the target-defining feature and the response feature were the same. That is, the detection of a digit also provided information about the correct response. For letter targets, on the other hand, the target-defining feature was the white color, and the response feature was the name of the white letter. The greater disparity between target-defining and response features for letter targets could have led to processing delays, resulting in poorer performance for the letters. A similar account has been given for the occurrence of illusory conjunctions in the time domain (see Botella, Barriopedro, \& Suero, 2001). Second, the distractors themselves were always letters, albeit black ones, but their inevitable processing to the level of recognition in most cases (e.g., Chun \& Potter, 1995) could have produced competitive interference in selecting and retaining the target letter's name (see also Isaak et al., 1999). Finally, it is possible that digits by themselves are more distinctive against a background of letters than are letters of different colors, making their presence easier to detect, at least in the present RSVP conditions.

Regardless of the cause or causes of the difference in $\mathrm{T} 1$ processing for letter or digit targets, the fact that $\mathrm{T} 1$ identification was easier for the numerals than for the letters should have produced a smaller AB for any target following a T1 numeral. This prediction is based on the assumption that ease of $\mathrm{T} 1$ processing determines how quickly attention can be reallocated to T2 processing following T1 completion (Seiffert \& Di Lollo, 1997). Consistent with this prediction, the $\mathrm{AB}$ effect, as measured by the reduction in accuracy in $\mathrm{T} 2$ report from lag 1 to lag 2, was twice as great following letter targets $(22 \%)$ than it was for digit targets $(11 \%)$ in Experiment 1 and $3 \%$ greater following letters $(12 \%)$ than after digits $(9 \%)$ in Experiment 2.

\section{Lag Effects}

The lag effect was different for T1 and T2, as is common in the AB literature. Although the T1 data are sometimes not included in published reports, the general finding is that $\mathrm{T} 1$ report accuracy is poorest at lag 1 - that is, if the two targets occur successively with no intervening distractors (Broadbent \& Broadbent, 1987; Peterson \& Juola, 2000). In the present Experiments 1A and 2, it was shown that much of the decrement in T1 report at lag 1 was due to order reversal errors, especially for NN trials. Not all of the T1 decrement at lag 1 could be explained by reversal errors alone, however. In some cases, there was an apparent loss in processing capacity, due to reallocation or sharing of resources when $\mathrm{T} 2$ followed $\mathrm{T} 1$ immediately.

The lag data for $\mathrm{T} 2$ report in Experiment 1 showed the U-shaped function characteristic in the traditional AB literature. That is, $\mathrm{T} 2$ report accuracy demonstrated the lag 1 sparing phenomenon described by Potter et al. (1998) and Visser et al. (1999). Performance tended to be worst at lag 2 or, sometimes, lag 3 and recovered after that to the level found at lag 1 . This effect was largely independent of target type and target blocking conditions, indicating the central nature of the capacity limitations that contribute to the AB (e.g., Chun \& Potter, 1995; Jolicœur et al., 2001).

\section{Location Effects}

In Experiment 2, location shifts between $\mathrm{T} 1$ and $\mathrm{T} 2$ eliminated the lag 1 sparing phenomenon, as was expected from previous research (Peterson \& Juola, 2000; Visser et al., 1999; but see also Shih, 2000). In Visser et al.'s terms, detection of T1 triggers an attentional episode that selects current information for detailed processing, leading to its consolidation into memory for later report. The temporal parameters of this selection process show sufficient spread to include not only the target item, but frequently the item following it in an RSVP stream. If the two targets are successive, both are likely to be included in the same attentional episode. Later items are not so selected, and if they include T2, it is likely to be missed unless $\mathrm{T} 1$ processing is complete by the time T2 appears. The spatial parameters of the selection process seem to be rather narrowly centered around $\mathrm{T} 1$, since even moving T2 horizontally by less than $2^{\circ}$ resulted in elimination of lag 1 sparing. This was true whether the participants knew the locations of T1 and T2 or not. That is, if the participants were prepared for $\mathrm{T} 1 \mathrm{on}$, say, the left 
side and $\mathrm{T} 2$ on the right, they apparently could not spread or divide the focus of attention over these two locations simultaneously. Rather, it was the case that either attention was focused at the expected location of $\mathrm{T} 1$ or T1's occurrence itself attracted the focus of attention, so that T2 report was much worse when it occurred less than $2^{\circ}$ away from $\mathrm{T} 1$, rather than at the same location. However, by lag 4 , the advantage for same-side targets disappeared, and there was even some evidence for a reversed advantage for different-side targets. This result is reminiscent of the inhibition-of-return phenomenon, and a similar reversal is apparent in the results reported by Shih (2000) in an experiment like our Experiment 2 (Figure 1A, p. 1351). Similar advantages for a T2 located away from the location of $\mathrm{T} 1$ have been reported recently in a task similar to the traditional $\mathrm{AB}$ paradigm, using multielement displays (Kristjánsson \& Nakayama, 2002).

\section{Task-Switching Costs}

In all the experiments, performance was worse for T1 if the two targets came from the same category (both letters or both digits), but performance on $\mathrm{T} 2$ was worse if they belonged to different categories. That is, a task repetition cost was observed for T1, and a task-switching cost was incurred for T2. Presenting two target items from the same category resulted in a loss in performance in identifying T1, and most (but not all) of this loss was due to reversal errors in the report of the two targets. Performance on T2, however, was decidedly worse if the targets came from different character sets, and this performance decrement was largest at lag 1 and decreased asymptotically at later lags (see also Rogers \& Monsell, 1995). Reconfiguration of the task set from detecting black digits to detecting white letters or vice versa entailed a cost of between $10 \%$ and $20 \%$ at lag 1 , which decreased to $5 \%$ or less by lag 6 . Chun and Potter (2001; see also Potter et al., 1998) asserted that the amount of lag 1 sparing is a sensitive index of task similarity between $\mathrm{T} 1$ and $\mathrm{T} 2$. In the present experiments, the categorical switch was not as devastating as the location switch for lag 1 sparing, a result consistent with the AB literature (Enns et al., 2001; Visser et al., 1999).

\section{Conclusions}

Many authors have lamented the fact that the $A B$ paradigm, and dual-task paradigms in general, have often confounded task-switching costs with other task demands in experimental tests of human temporal-processing abilities. In the AB task, any difference in definition of the two targets is likely to incur its own cost that is commingled with the AB effect itself. The present experiments are the first of which we are aware in which $A B$ performance with and without a concomitant task switch in a random mixture has been directly compared. In other conditions, task switch and no-switch trials were blocked in order to gauge the effectiveness of any preparation or reconfiguration required for dealing with identifying a white letter versus a black digit. In addition, Experiment 2 combined task switches with target location switches in the $A B$ paradigm, in an effort to determine whether the separate costs of these manipulations affect a common processing stage or, alternatively, separate levels of processing in visual attention and cognition.

We have found evidence for significant costs of both category shifts and location shifts in the identification of two targets presented in the RSVP format. Furthermore, these two effects are apparently independent and influence the AB phenomenon in different ways. Switching the target category from targets to letters and vice versa entailed a significant cost on $\mathrm{T} 2$ report, at least at short lags, and this effect was as large whether T1 and T2 were presented in the same location or were horizontally displaced from each other. The target category switch cost was also as large when the participants knew that the switch would occur between T1 and T2 as when the switch occurred randomly on half the trials. Either the costs of preparing for different target categories outweigh the benefits of using such a strategy, or the time needed to reconfigure target selection criteria is too great to achieve a measurable benefit in the RSVP task (Nieuwenhuis \& Monsell, 2002).

Since the task switch used in the present experiments primarily involved encoding operations only (letters vs. digits) without involving different response associations (the task in both instances was to remember the name of the target), it is likely that the task-switching costs were realized at relatively early levels of processing (Arrington, Altmann, \& Carr, 2003). That is, task-switching costs might involve central mechanisms if different, arbitrary stimulus-response association rules must be retrieved for a common stimulus set in both tasks (Monsell et al., 2003). The switching costs in the present experiments are likely to have been absorbed before the onset of a central bottleneck stage that is presumably dedicated to such tasks as memory consolidation and response selection (Arnell, 2001; Chun \& Potter, 2001; Ruthruff \& Pashler, 2001).

Switching location also had a large cost on T2 report probability at short lags, and again, this cost was independent of the participants' knowledge that a location switch would occur. There was no evidence of lag 1 sparing in our data, consistent with most studies of locationswitching effects in the AB paradigm. Location-shifting costs are likely to be incurred very early in visual processing, even before encoding is complete, since visual information is much more vulnerable to overwriting by subsequent inputs if attention is directed elsewhere (Enns et al., 2001). Furthermore, location shifting has its largest effect at lag 1 separation between the two targets, and the cost of a location switch is eliminated and even reversed at longer lags.

A notable exception to the present result has been reported in a study by Shih (2000), who found lag 1 sparing in a task similar to the one used here in Experiment 2. In Shih's task, the targets were always digits embedded 
in simultaneous, horizontally separated letter streams, and successive items appeared at a rather high rate of about 14 per second. Visser et al. (1999) commented that lag 1 sparing should be observable across location shifts if the spatial gradient of attention can encompass both streams. It is likely that participants did, in fact, attempt to attend to both streams in our task, as well as in Shih's, although both studies report an advantage for left-side targets, presumably due to an attentional bias toward the left. However, it is likely that the appearance of $\mathrm{T} 1 \mathrm{on}$ one side or the other constricts the spatial focus of attention momentarily while $\mathrm{T} 1$ is processed, resulting in a lag 1 deficit if T2 appears immediately after T1 in a different location. In Shih's study, the shorter SOA between successive items might have enabled some T2 processing to occur before narrowing of the spatial focus was complete, resulting in some amount of lag 1 sparing. Both our Experiment 2 and Shih's study show that lag 1 sparing is more closely associated with same-stream presentation for the two targets and that even a small shift in location results in a significant cost in $\mathrm{T} 2$ processing at short lags.

All of the experiments reported here demonstrated a significant loss in $\mathrm{T} 2$ processing for lags up to $400 \mathrm{msec}$ after $\mathrm{T} 1$ has been presented. This is the classic AB phenomenon. The fact that much of the $\mathrm{AB}$ deficit seems to be due to limitations in central memory processes, including memory consolidation, is supported by the fact that the $\mathrm{AB}$ effects we observed largely remained when combined with both location- and task-switching costs. That is, task switching diminished but did not eliminate the initial part of the U-shaped AB function, whereas location switching eliminated only lag 1 sparing. Both types of switches affected the AB phenomenon mainly at short lags. These results are consistent with those of others who have found independent effects of task switching and the AB (e.g., Allport \& Hsieh, 2001; Kawahara et al., 2003). In addition, the mutual independence of task-switching and location-switching effects that we observed echoes the observation that early selection of target features and target location are largely independent processes (e.g., Holmgren, 1968; Liu, Healey, \& Enns, 2003).

A summary model offered here that is consistent with the present results begins with specification of a search set for target-defining features present in one or two RSVP streams. In the present experiments, these included a white color or a digit, and the response feature was the identity of the target character. The detection of a target-defining feature signals a spatiotemporal focus of attention onto the item containing the feature (Botella et al., 2001). For T1, this usually results in a sufficient concentration of resources to select the target item, identify it, and execute central memory processes involved in consolidating a representation for later report. Report accuracy for $\mathrm{T} 1$ was $50 \%$ or better in most conditions, with the poorest levels of accuracy occurring when T1 and T2 were successive (lag 1), when T1 and T2 were both let- ters, and when the T1 location was unspecified in advance (Experiment 2).

Report of T2 was most accurate if it was presented at lag 1 (lag 1 sparing) or after two or three intervening items, if it was the same type of target as T1 (i.e., LL or $\mathrm{NN}$ trials) and if it occurred at the same location as T1. These results are consistent with the idea that the attentional episode triggered by the target-defining feature results in a spatiotemporal gradient that is more likely to include $\mathrm{T} 2$ if it matches $\mathrm{T} 1$ closely in features, location, and onset time. Otherwise, $\mathrm{T} 2$ processing is likely to be delayed, and while it is unattended, it is more vulnerable to masking and other forms of destructive interference from successive items than if it were selected for detailed processing. The present results indicate that each of these dimensions (the target-defining features, the target location, and its temporal relation to previous targets) independently affect coding and memory consolidation of T2. It appears that target location is important for spatial selection, with $100 \mathrm{msec}$ being a bit shorter than optimal for moving attention endogenously (Juola et al., 2000). Once spatial selection has occurred, target features are processed to determine which response code should be consolidated in memory. These processes are facilitated if they are compatible with the processes recently used to encode T1. If T2 belongs to a different category, taskswitching costs are incurred by delaying the time needed to encode T2 (Kawahara et al., 2003). Finally, the central processes involved in memory consolidation are largely serial in nature (Jolicœur et al., 2001), and the AB is most probably due to destructive loss of $\mathrm{T} 2$ information if it follows too closely on the heels of T1 but late enough to be excluded from the current attentional episode. The successive levels of processing location, feature, and categorical information presumably led to the observed independence of their effects in the present experiments on the $\mathrm{AB}$.

\section{REFERENCES}

Allport, A., \& Hsieh, S. (2001). Task-switching: Using RSVP methods to study an experimenter-cued shift of set. In K. Shapiro (Ed.), The limits of attention (pp. 36-64). New York: Oxford University Press.

Arnell, K. M. (2001). Cross-modal interactions in dual-task paradigms. In K. Shapiro (Ed.), The limits of attention (pp. 141-177). New York: Oxford University Press.

Arrington, C. M., Altmann, E. M., \& Carr, T. H. (2003). Tasks of a feather flock together: Similarity effects in task switching. Memory \& Cognition, 31, 781-789.

ARRINGTON, C. M., \& LOGAN, G. D. (2004). The cost of a voluntary task switch. Psychological Science, 15, 610-615.

Botella, J., BARriopedro, M. I., \& SUERo, M. (2001). A model of the formation of illusory conjunctions in the time domain. Journal of Experimental Psychology: Human Perception \& Performance, 27, 14521467.

Breitmeyer, B. (1984). Visual masking. Oxford: Oxford University Press.

Briand, K. A., \& KLeIN, R. M. (1987). Is Posner's "beam" the same as Treisman's "glue"? On the relation between visual orienting and feature integration theory. Journal of Experimental Psychology: Human Perception \& Performance, 13, 228-241. 
Broadbent, D. E., \& Broadbent, M. H. P. (1987). From detection to identification: Response to multiple targets in rapid serial visual presentation. Perception \& Psychophysics, 42, 105-113.

Chun, M. M., \& PotTer, M. C. (1995). A two-stage model for multiple target detection in rapid serial visual presentation. Journal of Experimental Psychology: Human Perception \& Performance, 21, 109-127.

CHUn, M. M., \& PotTer, M. C. (2001). The attentional blink and task switching within and between modalities. In K. Shapiro (Ed.), The limits of attention (pp. 20-35). New York: Oxford University Press.

Enns, J. T., Visser, T. A. W., Kawahara, J.-I., \& Di Lollo, V. (2001). Visual masking and task switching in the attentional blink. In K. Shapiro (Ed.), The limits of attention (pp. 65-81). New York: Oxford University Press.

Holmgren, J. (1968). Visual search with imperfect recognition. Perception \& Psychophysics, 4, 247-252.

IsAAK, M. I., ShaPIRO, K. L., \& MARTIN, J. (1999). The attentional blink reflects retrieval competition among multiple RSVP items: Tests of the interference model. Journal of Experimental Psychology: Human Perception \& Performance, 25, 1774-1792.

Jolicceur, P., Dell'AcQua, R., \& Crebolder, J. M. (2001). The attentional blink bottleneck. In K. Shapiro (Ed.), The limits of attention (pp. 82-99). New York: Oxford University Press.

Juola, J. F., Duvuru, P., \& Peterson, M. S. (2000). Priming effects in attentional gating. Memory \& Cognition, 28, 224-235.

Juola, J. F., Koshino, H., \& WarNer, C. B. (1995). Tradeoffs between attentional effects of spatial cues and abrupt onsets. Perception \& Psychophysics, 57, 333-342.

KaWAhara, J.-I., Zuvic, S. M., EnNs, J. T., \& Di Lollo, V. (2003). Task switching mediates the attentional blink even without backward masking. Perception \& Psychophysics, 65, 339-351.

Kristuánsson, Á., \& NAKAYAMA, K. (2002). The attentional blink in space and time. Vision Research, 42, 2039-2050.

Liu, G., Healey, C. G., \& EnNS, J. T. (2003). Target detection and localization in visual search: A dual systems perspective. Perception \& Psychophysics, 65, 678-694.

MEIRAN, N. (2000). Modeling cognitive control in task-switching. Psychological Research, 63, 234-249.

Monsell, S., Sumner, P., \& WATERS, H. (2003). Task-set reconfiguration with predictable and unpredictable task switches. Memory \& Cognition, 31, 327-342.

MÜLlER, H. J., \& RABBITT, P. M. A. (1989). Reflexive and voluntary orienting of visual attention: Time course of activation and resistance to interruption. Journal of Experimental Psychology: Human Perception \& Performance, 15, 315-330.

NiEUWENHUIS, S., \& MONSELL, S. (2002). Residual costs in task switching: Testing the failure-to-engage hypothesis. Psychonomic Bulletin \& Review, 9, 86-92.

Norman, D. A.., \& Shallice, T. (1986). Attention to action: Willed and automatic control of behavior. In R. J. Davidson, G. E. Schwartz, \& D. Shapiro (Eds.), Consciousness and self-regulation (Vol. 4, pp. 1-18). New York: Plenum.
Peterson, M. S., \& Juola, J. F. (2000). Evidence for distinct attentional bottlenecks in attention switching and attentional blink tasks. Journal of General Psychology, 127, 6-26.

PoSNER, M. I. (1980). Orienting of attention. Quarterly Journal of Experimental Psychology, 32, 3-25.

PoSNER, M. I., \& COHEN, Y. (1984). Components of visual orienting. In H. Bouma \& D. Bouwhuis (Eds.), Attention and performance X: Control of language processes (pp. 531-556). Hillsdale, NJ: Erlbaum.

Potter, M. C., Chun, M. M., Banks, B. S., \& Muckenhoupt, M. (1998). Two attentional deficits in serial target search: The visual attentional blink and an amodal task-switch deficit. Journal of Experimental Psychology: Learning, Memory, \& Cognition, 24, 979-992.

RaYMOND, J. E., ShaPIRo, K. L., \& ARNELl, K. M. (1992). Temporary suppression of visual processing in an RSVP task: An attentional blink? Journal of Experimental Psychology: Human Perception \& Performance, 18, 849-860.

ROGERS, R. D., \& MONSELL, S. (1995). Costs of a predictable switch between simple cognitive tasks. Journal of Experimental Psychology: General, 124, 207-231.

RUThrufF, E., \& PASHLER, H. E. (2001). Perceptual and central interference in dual-task performance. In K. Shapiro (Ed.), The limits of attention (pp. 100-123). New York: Oxford University Press.

Seiffert, A. E., \& Di Lollo, V. (1997). Low-level masking in the attentional blink. Journal of Experimental Psychology: Human Perception \& Performance, 23, 1061-1073.

SHAPIRO, K. L., \& RAYMOND, J. E. (1994). Temporal allocation of visual attention: Inhibition or interference? In D. Dagenbach \& T. H. Carr (Eds.), Inhibitory processes in attention, memory, and language (pp. 151-188). San Diego: Academic Press.

SHIH, S.-I (2000). Recall of two visual targets embedded in RSVP streams of distractors depends on their temporal and spatial relationship. Perception \& Psychophysics, 62, 1348-1355.

Sperling, G., \& WeichSelgartner, E. (1995). Episodic theory of the dynamics of spatial attention. Psychological Review, 102, 503-532.

TURVEY, M. T. (1973). On peripheral and central processes in vision: Inferences from an information-processing analysis of masking with patterned stimuli. Psychological Review, 80, 1-52.

Visser, T. A., \& BisCHOF, W. F. (2000, November). T1-difficulty and the attentional blink: We interrupt this processing to bring you an account. Paper presented at the 41st Annual Meeting of the Psychonomic Society, New Orleans.

Visser, T. A., Bischof, W. F., \& Di Lollo, V. (1999). Attentional switching in spatial and nonspatial domains: Evidence from the attentional blink. Psychological Bulletin, 125, 458-469.

Weichselgartner, E., \& Sperling, G. (1987). Dynamics of automatic and controlled visual attention. Science, 238, 778-780.

Wolfe, J. M. (1994). Guided Search 2.0: A revised model of visual search. Psychonomic Bulletin \& Review, 1, 202-238.

(Manuscript received March 5, 2003; revision accepted for publication January 21, 2004.) 